\title{
Overexpression of genes involved in phytochelatin biosynthesis in Escherichia coli: effects on growth, cadmium accumulation and thiol level ${ }^{*}$
}

\author{
Anna Wawrzyńska ${ }^{1}$, Adam Wawrzyński ${ }^{1}$, Dali Gaganidze ${ }^{2}$, Edyta Kopera ${ }^{1}$, \\ Katarzyna Piątek ${ }^{1}$, Wojciech Bal $^{1}$ and Agnieszka Sirko ${ }^{1 凶}$ \\ ${ }^{1}$ Institute of Biochemistry and Biophysics, Polish Academy of Sciences, Warszawa, Poland; \\ ${ }^{2}$ Scientific Research Center of Agriculture Biotechnology, Tibilisi 380062, Georgia
}

Received: 23 December, 2004; accepted: 18 February, 2005

Key words: cadmium, glutathione, phytochelatin, thiols

\begin{abstract}
In Escherichia coli, heterologous production of Schizosaccharomyces pombe phytochelatin synthase (PCS) along with overproduction of $E$. coli serine acetyltransferase (SAT) and $\gamma$-glutamylcysteine synthase $(\gamma \mathrm{ECS})$ was achieved and resulted in the accumulation of phytochelatins in bacterial cells. Overproduction of either $\gamma$ ECS alone or simultaneous production of all three proteins in bacterial cells were accompanied by reduced growth rate in liquid cultures. Interestingly, bacteria overproducing either $\gamma$ ECS or both SAT and $\gamma$ ECS (with elevated level of $\gamma$-glutamylcysteine but not of phytochelatins) were able to accumulate more cadmium per dry weight than the control. However, the most efficient cadmium accumulation was observed in bacteria with elevated levels of all three proteins: SAT, $\gamma$ ECS and PCS. Therefore, "pushing" the entire pathway might be the most promising approach in modification of bacteria for potential bioremediation purposes because the level of intermediates, cysteine and glutathione, can limit the rate of production of phytochelatins. However, in such bacteria other metabolic process might become limiting for efficient growth.
\end{abstract}

\footnotetext{
This work was partially supported by the EU Commission through funding of FP5 project PHYTAC (QLRT-2001-00429 and QLRT- 2001-02778 [NAS]). D.G. was a holder of a fellowship from the European Fellowships Fund at IBB PAS.

${ }^{凶}$ Correspondence to: A. Sirko, Institute of Biochemistry and Biophysics, Polish Academy of Sciences, A. Pawińskiego 5A, 02-106 Warszawa, Poland; e-mail: asirko@ibb.waw.pl

Abbreviations: AP, alkaline phosphatase; CMQT, 2-chloro-1-methylquinolinium tetrafluoroborate; $\gamma$-Glu-Cys, $\gamma$-glutamylcysteine; $\gamma \mathrm{ECS}, \gamma$-glutamylcysteine synthase; GSH, glutatione; IPTG, isopropyl $\beta$-D-1-thiogalactopyranoside; ORF, open reading frame; PBS, phosphate-buffered saline; PC, phytochelatin; PCS, phytochelatins synthase; rbs, ribosome-binding site; SAT, serine acetyltransferase; TFA, trifluoroacetic acid.
} 
Organisms can avoid toxicity of heavy metals by three mechanisms: diminished accumulation, detoxification/reduction or complexation with ligands. An important class of heavy metal binding ligands is a family of phytochelatins (PCs), peptides that consist of repetitions of the $\gamma$-glutamylcysteine $(\gamma$-GluCys) dipeptide followed by a terminal glycine - the basic structure being ( $\gamma$-Glu-Cys) $)_{\mathrm{n}}$-Gly $\left[(\mathrm{PC})_{n}\right]$, where $\mathrm{n}$ is generally in the range two to seven. PCs have been identified in a wide variety of plant species and in some microorganisms. The enzyme responsible for their production, phytochelatin synthase (PCS), was first identified by Grill et al. (1989) and characterized in a number of subsequent studies (Chen et al., 1997; Klapheck et al., 1995). The enzyme is a $\gamma$-Glu-Cys dipeptidyl transpeptidase and catalyses transpeptidation of the $\gamma$-Glu-Cys moiety of GSH either onto a second glutathione (GSH) molecule to form $\mathrm{PC}_{(\mathrm{n}=2)}$ or onto a $\mathrm{PC}$ molecule to produce a $\mathrm{PC}_{(\mathrm{n}+1)}$ oligomer.

Significant recent advances in understanding of the molecular basis of PC biosynthesis and function have resulted from molecular genetic studies conducted in a number of model organisms. These approaches centered on the identification of Cd-sensitive mutants of the plant Arabidopsis thaliana and the yeasts Schizosaccharomyces pombe and Candida glabrata. In addition, expression of plant cDNAs in Escherichia coli and Saccharomyces cerevisiae cells was particularly useful in the identification and analysis of genes involved in functions related to heavy metal detoxification. PC biosynthesis may be regulated by a number of mechanisms. For example, in Brassica juncea, exposure to $\mathrm{Cd}$ produces a requirement for both cysteine and GSH for PC biosynthesis that is met by coordinate transcriptional regulation of genes involved in sulfur transport and assimilation (Heiss et al., 1999; Lee \& Leustek, 1999) as well as GSH biosynthesis (Schafer et al., 1998). The prominent function of $\gamma$-Glu-Cys peptides is sequestration of metals. It results in creation of metal-containing complexes that are accretions of multiple peptides of various lengths with metal ions.

The PC synthase (PCS) activity is thought to be the major determinant of the rate of $\mathrm{PC}$ synthesis. Kinetic studies using plant cell cultures exposed to $\mathrm{Cd}$ demonstrated that $\mathrm{PC}$ biosynthesis occurs within minutes of exposure to the heavy metal and is independent of de novo protein synthesis (Rauser, 1999). PCS is activated by $\mathrm{Cd}^{2+}, \mathrm{Cu}^{2+}, \mathrm{Ag}^{+}, \mathrm{Hg}^{2+}$, $\mathrm{Zn}^{2+}$ and $\mathrm{Pb}^{2+}$ ions (Chen et al., 1997; Klapheck et al., 1995). The mechanism by which PCS is activated appears to be relatively non-specific with respect to the activating metal ion, although some metals are more effective than others. Overproduction of PCS from wheat in $S$. cerevisiae enhances tolerance to and accumulation of cadmium (Clemens et al., 1999). Recently, E. coli cells expressing A. thaliana PCS gene showed a marked accumulation of phytochelatins that in turn caused, after exposure, accumulation of heavy metals such as cadmium or metalloid arsenic (Sauge-Merle et al., 2003). The authors observed a rapid depletion of glutathione in these bacteria. This result is in agreement with the observation that transgenic Arabidopsis overexpressing an Arabidopsis phytochelatin synthase gene (AtPCS1) showed, paradoxically, hypersensitivity to $\mathrm{Cd}$ or Zn (Lee et al., 2003), which disappeared when GSH was supplemented in the medium. Thus, the level of the substrate for PCS, GSH-Cd, might be the limiting factor determining the rate of phytochelatin synthesis. It is also possible that the antioxidative system in plants might have been affected by overexpression of AtPCS1 and this may have resulted in the observed hypersensitivity. The oxidative stress might have been generated in those plants by cadmium, as its phytotoxicity is generally ascribed to its reactivity with ligands containing oxygen, nitrogen and sulfur that often produce reactive oxygen species (Sanita di Toppi \& Gabbrielli, 1999). Although this clearly demonstrates that PCs 
can have an important role in detoxification, it remains to be determined whether this is their primary or incidental function. Other proposed roles for PCs include their involvement in essential heavy metal homeostasis, iron metabolism and sulfur metabolism (Rauser, 1999; Zenk, 1996).

The aim of this work was to investigate the effects of simultaneous overproduction in $E$. coli cells of three enzymes involved in phytochelatin biosynthesis: PCS from $S$. pombe and two proteins from $E$. coli, serine acetyltransferase (SAT) and $\gamma$-glutamylcysteine synthase ( $\gamma$ ECS). SAT is involved in the production of $O$-acetylserine and is supposed to limit the synthesis of cysteine, while $\gamma \mathrm{ECS}$ is involved in the first step of glutathione synthesis assumed to be much more limiting than the second step catalyzed by glutathione synthase.

\section{MATERIALS AND METHODS}

\section{Bacterial growth and culture condi-} tions. E. coli strain BL21(DE3) was transformed with plasmids indicated: pET28a, pGCP, pGCE or pGSH. Bacteria were grown in LB medium in the presence of kanamycin ( $50 \mu \mathrm{g} / \mathrm{ml}$ ). $\mathrm{O} / \mathrm{N}$ cultures were usually grown at $37^{\circ} \mathrm{C}$, then refreshed $(1: 100)$ in the same medium and grown at $30^{\circ} \mathrm{C}$ with rotary shaking. $\beta$-D-1-thiogalactopyranoside (IPTG) was added to the cultures at $\mathrm{A}_{600} 0.3-0.5$ to a final concentration of $1 \mathrm{mM}$. After one hour of induction, $\mathrm{CdCl}_{2}$ was added to the concentrations indicated. Cadmium content in bacteria was measured always in cultures grown for 5 $\mathrm{h}$ in the presence of $0.5 \mathrm{mM} \mathrm{CdCl} 2$.

DNA cloning and plasmid construction. Standard molecular cloning techniques were performed as described (Sambrook et al., 1989). The following oligonucleotides were used for amplification of the $E$. coli cysE (EXEC1: 5'-cgt cta gaa agg aga tat acc atg tcg tgt gaa gaa ctg gaa a-3' and EXEC2: $5^{\prime}$-gct cta gag tcg act tag atc cca tcc cca tact ca-3') and
gshA (GSH1: 5'-cgg gat cca tgg tcc cgg acg tat ca gag-3' and GSH2: $5^{\prime}$-aac tgc agt cta gat cag geg tgt ttt tcc ag-3') and S. pombe PCS (EXPC1: $5^{\prime}$-cgg tcg aca agg aga tat acc atg gac att gtt aaa cga gca gtc-3' and EXPC2: 5'-gcg tcg act cac gta ttt tta cag cag ctt ga- $3^{\prime}$ ) genes using the respective genomic DNA.

Western blots. Proteins were resolved in SDS/polyacrylamide $12.5 \%$ gels. Electrophoresis was carried out using conditions recommended by the manufacturer of the electrophoresis apparatus (BioRad). The separated proteins were electroblotted onto either nitrocellulose membrane (Amersham) or PVDF membrane (Schleicher \& Schuell) using an apparatus for "wet transfer" (Kucharczyk T.E., Poland) following the manufacturer's protocol. Membranes carrying immobilized proteins were incubated for $1 \mathrm{~h}$ in a blocking solution (5\% non-fat milk in PBS (10 $\mathrm{mM} \mathrm{Na} / \mathrm{KPO}_{4}$ buffer, $0.15 \mathrm{M} \mathrm{NaCl}, \mathrm{pH}$ 7.4)) to saturate non-specific binding sites. Then they were incubated for $1 \mathrm{~h}$ in a solution of specific rabbit polyclonal antibody (primary antibody) diluted in PBS, followed by four washes (5 min each) in PBS. As a secondary antibody, anti-rabbit IgG conjugated with alkaline phosphatase (AP) were used (Sigma). The 60 min incubation with secondary antibody was followed by four washes $(5 \mathrm{~min}$ each) in PBS and finally, NBT/BCIP (Promega) was used as the substrate for the AP enzymatic reaction.

Determination of thiols in bacterial samples. Bacteria from $100 \mathrm{ml}$ cultures were centrifuged $\left(8000 \times \boldsymbol{g}, 10 \mathrm{~min}, 4^{\circ} \mathrm{C}\right)$, washed in $0.9 \% \mathrm{NaCl}$, resuspended in $500 \mu \mathrm{l}$ of $0.1 \mathrm{M}$ $\mathrm{HCl}$, sonicated 3 times ( $5 \mathrm{~min}$ each) and centrifuged $\left(14000 \times \mathbf{g}, 15 \mathrm{~min}, 4^{\circ} \mathrm{C}\right)$ to remove debris. To $200 \mu \mathrm{l}$ of the extract, $28 \mu \mathrm{l}$ of $1 \mathrm{M}$ $\mathrm{NaOH}$ and $8 \mu \mathrm{l}$ of $6 \mathrm{M} \mathrm{NaBH}_{4}$ was added. The mixture was vortexed and stored for $2 \mathrm{~min}$. Then, $20 \mu \mathrm{l}$ of $3 \mathrm{M} \mathrm{HCl}$ was added in order to decompose the excess of $\mathrm{NaBH}_{4}$. The mixture was centrifuged $(14000 \times \mathbf{g}, 1 \mathrm{~min})$. Next, $400 \mu \mathrm{l}$ of $0.2 \mathrm{M}$ phosphate buffer, $\mathrm{pH} 7.6$, and $10 \mu \mathrm{l}$ of $0.1 \mathrm{M}$ 2-chloro-1-methylquinolinium 
tetrafluoroborate (CMQT) was added, the mixture was vortexed, put aside for $4 \mathrm{~min}$, and acidified by adding $100 \mu \mathrm{l}$ of $3 \mathrm{M} \mathrm{HCl}$, followed by centrifugation $(12000 \times \boldsymbol{g}, 3 \mathrm{~min})$ and filtration through a $0.22 \mu \mathrm{m}$ filter. Aliquots of $50 \mu \mathrm{l}$ of the final solution were injected into an HPLC system (Waters Breeze) via an autosampler and separated on a C-18 column (Waters Symmetry, $4.6 \times 150 \mathrm{~mm}$ ). Elution buffer A was $0.1 \%(\mathrm{v} / \mathrm{v})$ trifluoroacetic acid (TFA) in $\mathrm{H}_{2} \mathrm{O}$ and buffer $\mathrm{B}$ was $90 \%(\mathrm{v} / \mathrm{v})$ acetonitrile in $\mathrm{H}_{2} \mathrm{O}, 0.1 \%$ TFA. The gradient used for these experiments was $0-50 \%$ buffer $\mathrm{B}$ in $25 \mathrm{~min}$, flow rate $1 \mathrm{ml} / \mathrm{min}$, detection by absorption at $354 \mathrm{~nm}$.

Determination of cadmium in bacterial cells. Dried samples (bacterial cells), after suspending in $9 \mathrm{ml}$ of $65 \% \mathrm{HNO}_{3}$ supplemented with $0.9 \mathrm{ml} 35 \% \mathrm{H}_{2} \mathrm{O}_{2}$, were mineralized for $25 \min \left(180^{\circ} \mathrm{C}, 942 \mathrm{~W}\right)$ in a laboratory microwave oven ETHOS PLUS (Milestone). Then the samples were cooled and $0.1 \mathrm{ml}$ of $35 \% \mathrm{H}_{2} \mathrm{O}_{2}$ was added. $\mathrm{Cd}$ content in bacterial cells was measured using Flame Atomic Absorption Spectrophotometry (SOLAAR M6, TJA Solutions), mixture of air/acetylene, absorption at $228.8 \mathrm{~nm}$. The samples were measured in triplicates.

\section{RESULTS}

\section{Construction of plasmids containing genes involved in phytochelatin biosynthesis}

Plasmids containing cys $E$ and gsh $A$ genes from $E$. coli or PCS from $S$. pombe previously cloned in our laboratory (Błaszczyk, 2004) served as templates in polymerase chain reaction (PCR). The pairs of primers EXEC1/ EXEC2, GSH1/GSH2 and EXPC1/EXPC2 were used to obtain cysE, gshA and PCS open reading frame (ORF) fragments, respectively. The PCR products $(0.8 \mathrm{~kb}$ for cysE, $1.6 \mathrm{~kb}$ for gsh $A$ and $1.2 \mathrm{~kb}$ for PCS) were digested with appropriate restriction enzymes, cloned separately into pUC19 and sequenced. Sequencing confirmed the presence of the respective ORFs without errors. The subsequent multistep cloning into pET28a vector resulted in the construction of the plasmids schematically shown in Fig. 1. Plasmid pGSH contains gshA gene transcribed as a monocistronic transcript from a T7 polymerase-dependent

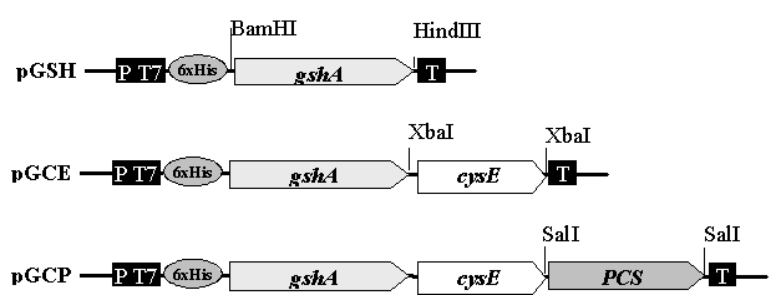

Figure 1. Schematic map of the inserts present in the plasmids used in this work.

The vector in all cases is pET28a. Only the restriction sites used for final cloning are shown. PT7, promoter of T7 bacteriophage; $6 \mathrm{x}$ His, histidine tag; T, transcription terminator; gsh $A$, cysE and PCS, sequences encoding $E$. coli $\gamma$-glutamylcysteine synthase ( $\gamma \mathrm{ECS})$, serine acetyltransferase (SAT) and $S$. pombe phytochelatin synthase (PCS), respectively.

promoter. Polycistronic transcripts can be formed in the case of pGCE and pGCP containing gshA/cysE and gshA/cysE/PCS, respectively. Efficient translation of each ORF is assured by separate ribosome-binding sites (rbs) introduced upstream of the coding regions of cysE and PCS, while gshA can be translated using the rbs present in pET28a.

Comparison of growth rates, accumulation of cadmium and the level of non-protein thiols in bacteria transformed with the constructed plasmids

Plasmids pET28a, pGCP, pGCE or pGSH were introduced into $E$. coli and the transformants were grown in LB medium containing $0.5 \mathrm{mM} \mathrm{CdCl} 2$ in order to identify the plasmid causing the strongest cadmium accumulation in bacterial cells. The growth of the bacterial cultures was monitored by measurement of absorbance at $600 \mathrm{~nm}$ (Fig. 2B) and 
cadmium accumulation was assayed and calculated per mg of dry mass of bacterial cells (Fig. 2C). In parallel, cultures were grown in the LB medium without cadmium (Fig. 2A).
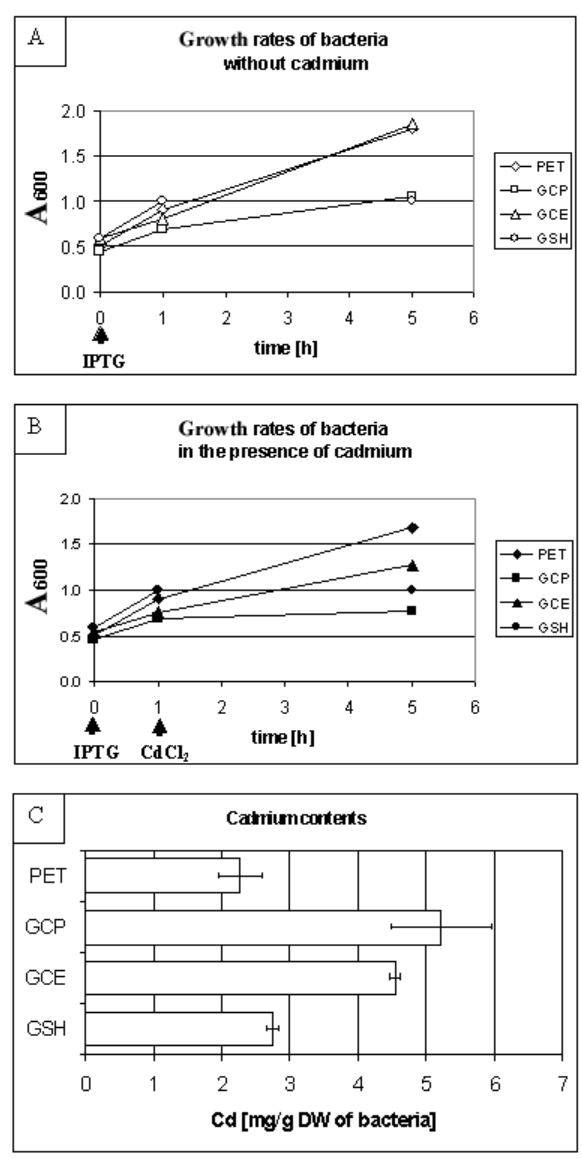

Figure 2. Growth rates of $E$. coli transformants at $30^{\circ} \mathrm{C}$ in $\mathrm{LB}$ medium without (A) and with $0.5 \mathrm{mM}$ $\mathrm{CdCl}_{2}$ (B), and cadmium contents in dry weight (DW) of bacteria (C).

For the growth rates, one representative of three independent cultures is shown with arrows indicating the time of addition of IPTG and $\mathrm{CdCl}_{2}$. For the cadmium contents, average values from three independent cultures (each measured in triplicate) are shown with standard deviations indicated. PET, pET28a (vector); GCP, pGCP (cysE gshA PCS); GCE, pGCE (cysE gshA); GSH, pGSH (gshA).

The results indicated that bacteria harboring pGCP accumulated more cadmium than the other transformants, next were bacteria transformed with pGCE and pGSH, the latter being only a bit more effective than the bacteria transformed with pET28a. The growth of the transformants in the medium containing cadmium varied depending on the plasmid, which can be ordered as follow: pGCP, pGSH, pGCE and pET28a from the slowest to the best growers (Fig. 2B). Surprisingly, overproduction of either $\gamma$ ECS alone or of all three proteins resulted in growth retardation, even without cadmium in the medium. Overproduction of SAT together with $\gamma$ ECS resulted in a growth rate similar to the control.

To monitor the effects of the overproduction of the proteins in question on the levels of non-protein thiols, acid extracts from bacterial cells were analyzed by HPLC after derivatization of $\mathrm{SH}$-groups with CMQT (Fig. 3). The identity of the marked peaks

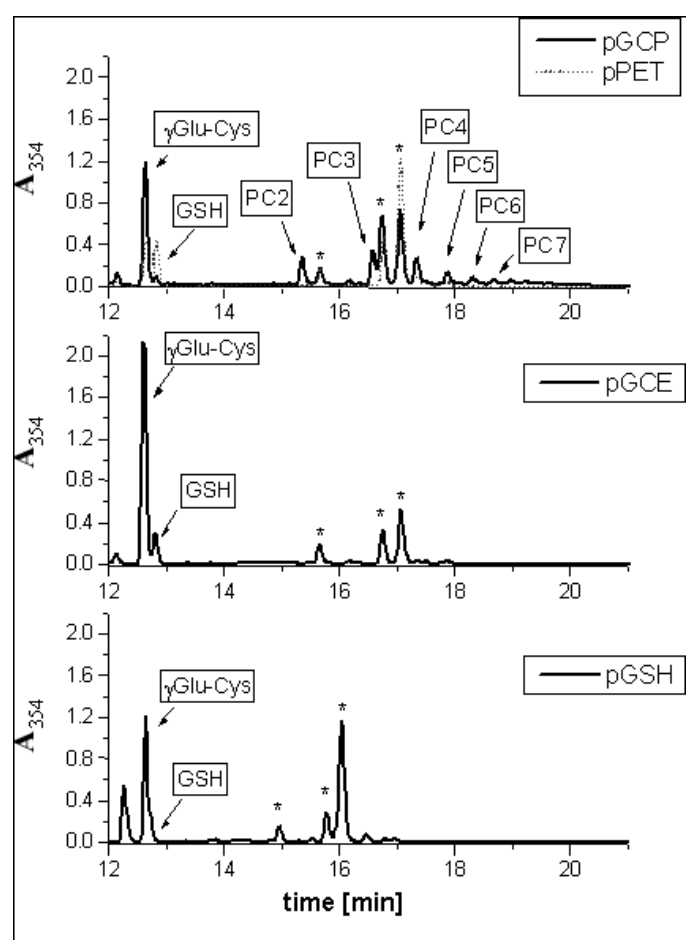

Figure 3. HPLC chromatograms of acidic extracts of bacterial samples.

Peaks due to non-thiol compounds are marked with (*). Positions of identified thiols are indicated. The bacteria strains are transformed with the plasmids indicated.

( $\gamma$ Glu-Cys, GSH, PC2, PC3, PC4, PC5, PC6, PC7) was confirmed by mass-spectroscopy analysis (not shown). The results clearly demonstrate that (i) phytochelatins are produced in bacteria transformed with pGCP, but not in those transformed with the other plasmids, 
(ii) GSH level in these bacteria is lower than in the other strains, (iii) in bacteria transformed with plasmid pGCE containing two genes - cys $E$ and gsh $A$ - a strong increase of $\gamma$ Glu-Cys level is observed, and (iv) a much smaller elevation of $\gamma$ Glu-Cys level is observed in bacteria transformed with pGSH.

Detection of the proteins encoded by the polycistronic transcript of the pGCP plasmid

A polycistronic transcript was expected to be formed upon induction of the T7 polymerase-dependent promoter of the plasmid pGCP. To test if efficient translation of all open reading frames took place, expression of each of the three genes was verified at the protein level by Western blot technique using previously obtained three types of rabbit polyclonal antibodies specifically recognising $E$. coli SAT, $\gamma \mathrm{ECS}$ and $S$. pombe PCS (Błaszczyk, 2004). The genes cysE (encoding SAT) and gsh $A$ (encoding $\gamma \mathrm{ECS}$ ) are present in the $E$. coli genome; however, in the conditions of the experiment no SAT or $\gamma$ ECS proteins were detected in control bacteria transformed with the empty plasmid pET28a (Fig. 4C). This is probably due to the low expression of these genes in the rich medium. Concerning the expression from the plasmid pGCP, PCS as well as $\gamma$ ECS were expressed quite efficiently, while only a low level of SAT protein was found (Fig. 4C). The level of the proteins was apparently unaffected by the presence of cadmium in the medium.

\section{DISCUSSION}

The growth characteristics of bacteria overproducing SAT, $\gamma$ ECS and PCS in various combinations confirm that efficient cysteine production might be the limiting factor for glutathione biosynthesis in bacteria (Li et al., 2004). This would explain the growth reduction of the strain containing pGSH plasmid
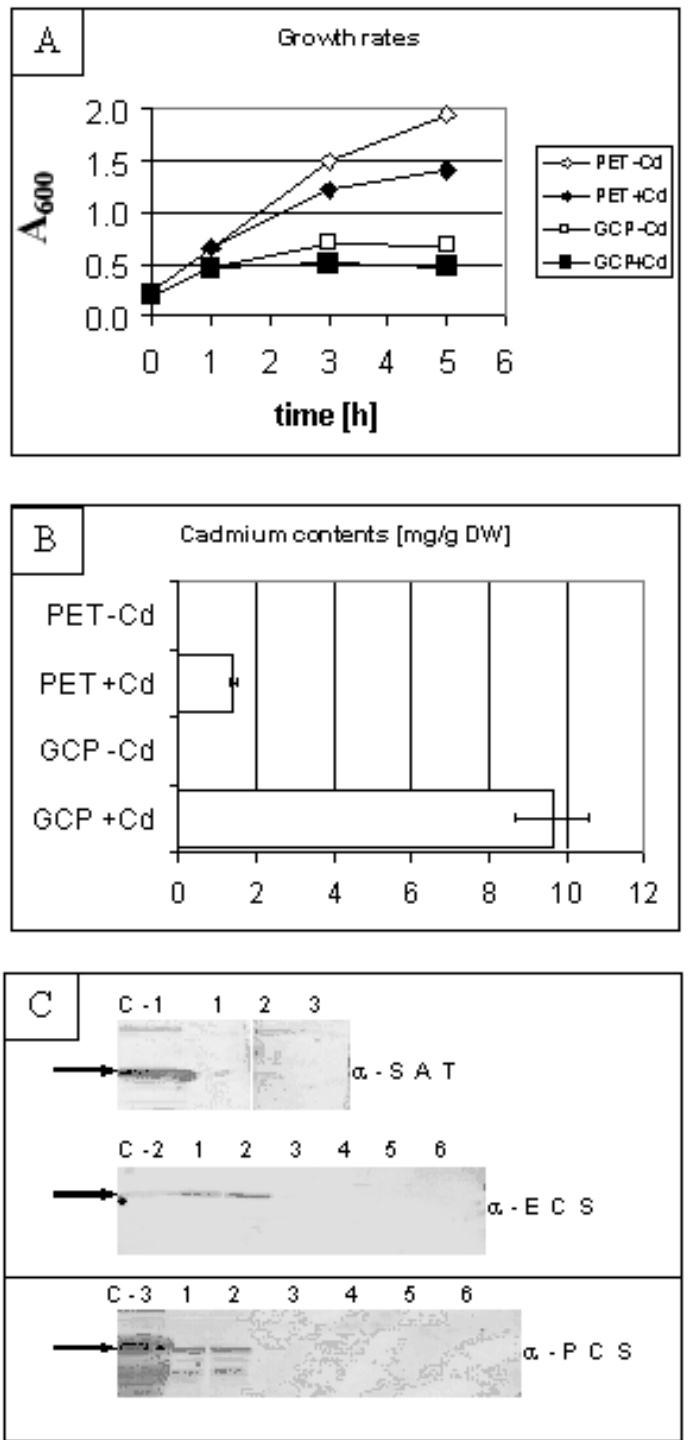

Figure 4. Selected characteristics of $E$. coli transformed with pGCP (GCP) and pET28a (PET) grown at $30^{\circ} \mathrm{C}$ in $\mathrm{LB}$ medium with 0 or $0.5 \mathrm{mM}$ $\mathrm{CdCl}_{2}$.

Panel A. Comparison of growth rates; Panel B. Cadmium accumulation in the dry weight (DW) of bacteria; Panel C. Western blot of protein extracts from the bacterial transformants using rabbit polyclonal antibodies as indicated. C-1, C-2 and C-3 are extracts from transformants containing appropriate plasmids with single genes, cysE, gsh $A$ and PCS, respectively. Protein extract from bacteria containing: 1 , pGCP, grown with IPTG in the presence of $0.5 \mathrm{mM} \mathrm{CdCl}_{2} ; 2$, pGCP, grown with IPTG without cadmium; 3, pGCP, without IPTG and without cadmium; 4, pET28a, with IPTG and 0.5 $\mathrm{mM} \mathrm{CdCl}_{2} ; 5$, pET28a, with IPTG without cadmium; 6 , pET28a, without IPTG and without cadmium.

regardless of the presence of cadmium. An alternative explanation, that the reduced 
growth of bacteria overproducing $\gamma \mathrm{ECS}$ is a result of ATP depletion (Li et al., 2004) can be ruled out because additional overproduction of SAT not only improves the growth of bacteria with higher $\gamma$ ECS level (Fig. 2A, B) but also results in a higher level of $\gamma$ Glu-Cys. Interestingly, the GSH level in these bacteria is not increased in comparison to the control (Fig. 3). The elevation of $\gamma$ Glu-Cys was probably responsible for the increased accumulation of cadmium by bacteria overproducing SAT and $\gamma$ ECS (pGCE) and bacteria overproducing $\gamma \mathrm{ECS}$ alone (pGSH).

Simultaneous overproduction of all three proteins resulted in a decrease of glutathione in comparison to all other strains. As the level of $\gamma$ Glu-Cys was higher in this strain than in the control, this observation suggests that the next enzymatic activity, glutathione synthase, was limiting for production of phytochelatins.

In summary, bacteria producing phytochelatins due to inducible expression of three genes: $S$. pombe PCS and E. coli cysE and $g \operatorname{sh} A$ were obtained. The important observation was that transformants overproducing all three proteins grew slower than the control in medium without cadmium (however, cadmium additionally retarded their growth). Then, overproduction of these proteins did not improve bacterial tolerance to cadmium but resulted in increased cadmium accumulation per dry weight of bacteria. Interestingly, in the analyzed bacteria not only phytochelatins and GSH but apparently also $\gamma$ Glu-Cys is involved in binding of cadmium ions.

We gratefully acknowledge Dr. Grazyna Chwatko and Professor Edward Bald from the Department of Enviromental Chemistry, University of Łódź (Poland) for providing us with a batch of 2-chloro-1-methylquinolinium tetrafluoroborate (CMQT) and for sharing their expertise regarding application of CMQT in the HPLC analysis of thiols. We are grateful to Professor Aleksandra
Skłodowska, Laboratory of Environmental Pollution Analysis, Faculty of Biology, Warsaw University (Poland) for performing the assays of cadmium.

\section{R E F E R E N C E S}

Błaszczyk A. (2004) Modification of sulfur metabolism in tobacco and identification of genes regulated by sulfur starvation. $\mathrm{PhD}$ Thesis, Institute of Biochemistry and Biophysics, Polish Academy of Sciences, Warsaw.

Chen J, Zhou J, Goldsbrough PB. (1997) Characterization of phytochelatins synthase from tomato. Physiol Plant.; 101: 165-72.

Clemens S, Kim EJ, Neumann D, Schroeder JI. (1999) Tolerance to toxic metals by a gene family of phytochelatin synthases from plants and yeast. EMBO J.; 18: 3325-33.

Grill E, Loffler S, Winnacker E-L, Zenk M. (1989) Phytochelatins, the heavy metal binding peptides of plants, are synthesized from glutathione by a specific gamma-glutamylcysteine dipeptidyl transpeptidase (phytochelatins synthase). Proc Natl Acad Sci USA.; 86: 6838-42.

Heiss S, Schafer HJ, Haag-Kerwer A, Rausch T. (1999) Cloning sulfur assimilation genes of Brassica juncea L.: cadmium differentially affects the expression of a putative low-affinity sulfate transporter and isoforms of ATP sulfurylase and APS reductase. Plant Mol Biol.; 39: 847-57.

Klapheck S, Schlunz S, Bergmann L. (1995) Synthesis of phytochelatins and homo-phytochelatins in Pisum sativum L. Plant Physiol.; 107: 515-21.

Lee M, Leustek T. (1999) Identification of the gene encoding homoserine kinase from Arabidopsis thaliana and characterization of the recombinant enzyme derived from the gene. Arch Biochem Biophys.; 372: 135-42.

Lee S, Moon JS, Ko TS, Petros D, Goldsbrough PB, Korban SS. (2003) Overexpression of Arabidopsis phytochelatin synthase paradoxi- 
cally leads to hypersensitivity to cadmium stress. Plant Physiol.; 131: 656-63.

Li Y, Wei G, Chen J. (2004) Glutathione: a review on biotechnological production. Appl Microbiol Biotechnol.; 66: 233-42.

Rauser WE. (1999) Structure and function of metal chelators produced by plants: the case for organic acids, amino acids, phytin, and metallothioneins. Cell Biochem Biophys.; 31: $19-48$.

Sambrook J, Fritsch EF, Maniatis T. (1989) Molecular Cloning: A Laboratory Manual. Cold Spring Harbor, Cold Spring Harbor Laboratory Press.

Sanita di Toppi LS, Gabbrielli R. (1999) Response to cadmium in higher plants. Environ Exp Bot.; 41: 105-30.
Sauge-Merle S, Cuine S, Carrier P, Lecomte-Pradines C, Luu DT, Peltier G. (2003) Enhanced toxic metal accumulation in engineered bacterial cells expressing Arabidopsis thaliana phytochelatin synthase. Appl Environ Microbiol.; 69: 490-4.

Schafer HJ, Haag-Kerwer A, Rausch T. (1998) cDNA cloning and expression analysis of genes encoding GSH synthesis in roots of the heavy-metal accumulator Brassica juncea L.: evidence for $\mathrm{Cd}$-induction of a putative mitochondrial gamma-glutamylcysteine synthetase isoform. Plant Mol Biol.; 37: 87-97.

Zenk MH. (1996) Heavy metal detoxification in higher plants - a review. Gene.; 179: 21-30. 